\title{
Energy Harvesting and Management for Wireless Autonomous Sensors
}

\author{
Alex S. Weddell, Geoff V. Merrett, Nick R. Harris, and Bashir M. Al-Hashimi \\ Pervasive Systems Centre, ECS, University of Southampton, SO17 1BJ, UK \\ +44 (0)23 8059 4996, \{asw05r, gm04r, nrh, bmah\}@ecs.soton.ac.uk
}

\begin{abstract}
Executive Summary: Wireless autonomous sensors that harvest ambient energy are attractive solutions, due to their convenience and economic benefits. A number of wireless autonomous sensor platforms which consume less than $100 \mu \mathrm{W}$ under dutycycled operation are available. Energy harvesting technology (including photovoltaics, vibration harvesters, and thermoelectrics) can be used to power autonomous sensors. A developed system is presented that uses a photovoltaic module to efficiently charge a supercapacitor, which in turn provides energy to a microcontroller-based autonomous sensing platform. The embedded software on the node is structured around a framework in which equal precedent is given to each aspect of the sensor node through the inclusion of distinct software stacks for energy management and sensor processing. This promotes structured and modular design, allowing for efficient code reuse and encourages the standardisation of interchangeable protocols.
\end{abstract}

\section{INTRODUCTION}

Wireless autonomous sensors are devices with sensing, processing and wireless communication capabilities, able to act without assistance from other devices. Wireless sensing is attractive as the cost of wiring generally exceeds the cost of sensors, with a study putting the cost of providing wiring to a single conventional sensor in a commercial building at $\$ 200^{l}$. The high installation cost for wired sensors, along with the need to embed sensors in complex machinery and inaccessible locations, has spurred recent developments in autonomous sensing and wireless sensor networking. The success of such systems is dependent on the longevity of sensor nodes and the quality of information they can provide.

By definition, wireless autonomous sensors must not depend on an external power supply. Conventionally, such devices have been powered by primary (non-rechargeable) batteries which are replaced when depleted. Recent progress in the development of energy harvesting technologies, scavenging electrical energy from light, vibration, or temperature difference, now permits sensors to be free from the constraints of operation from primary batteries.

In order to effectively use harvested electrical energy, it must be 'buffered' in capacitors or secondary (rechargeable) batteries, and the autonomous sensor must have an awareness of its energy status (so that it can control its activity based on its available energy). Additionally, energy harvesting devices are sensitive to their operating conditions, and it may be necessary to adapt the load they are subjected to in order to improve the efficiency of the system. Furthermore, the complex behaviour of energy sources, stores and consumers means that achieving true energy awareness is non-trivial.

In this paper, we review the current state-of-the-art for energy harvesting autonomous sensors, and explore some of the issues related to energy management. A number of commercially-available autonomous sensor platforms are introduced, along with energy harvesting and storage devices. Three examples of energy-harvesting 
autonomous sensors are described in depth, and an autonomous sensor system that has been developed in-house and which is powered from indoor lighting is introduced. We describe the hardware, and novel software structure, of the system in detail.

\section{AUTONOMOUS SENSOR PLATFORMS}

A number of wireless sensor platforms have been developed and commercialised, each having different capabilities and features such as on-board sensors. Berkeley motes have been commercialised by Crossbow, and have achieved dominance through their flexible interfacing, small size and reasonable cost. Used by over 100 research organisations, the motes are normally used with TinyOS (an embedded operating system for wireless sensors) and are available in a number of incarnations with a range of sensor boards available. The mote is pitched as a generic sensing platform, with moderate capabilities for sensing, data processing and communications. Many users, however, find the TinyOS system to be restrictive and inefficient ${ }^{2}$.

Various other platforms are available, some incorporating a radio transceiver and microprocessor in a systemon-chip. The TI/Chipcon CC2430 (shown in Figure 1) features an enhanced 8051 microprocessor and an IEEE 802.15.4-compliant radio transceiver operating in the $2.4 \mathrm{GHz}$ ISM band ${ }^{3}$. Microchip have produced a transceiver which is compatible with their range of microcontrollers ${ }^{4}$.

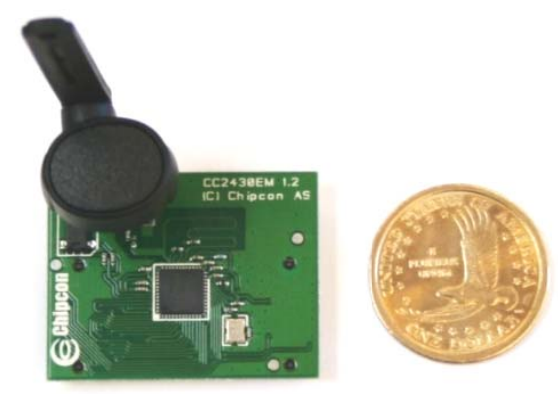

Figure 1: A CC2430 sensor node (power supply not shown)

In highly energy-constrained applications, it is important to exercise detailed control over the operation of the system. The embedded software for sensor nodes is generally written in a variant of ANSI C, with the option to code aspects of the system in assembler. Platform manufacturers will normally provide the communication stack software for their device (for free, in the case of TI/Chipcon and Microchip, although some depend on commercial compilers), leaving it to the system developer to tailor the software to the application.

In order to extend the system lifetimes, platforms will normally spend most of their time in a very low power 'sleep' mode, being woken up by an internal timer or external interrupt. The sleep power consumption of these devices is generally very low, typically around $1 \mu \mathrm{W}$. This low sleep power consumption, along with a low duty cycle of operation, means that the energy requirements of autonomous sensor platforms can be very low indeed. Table 1 shows the typical energy requirements of a CC2430 in its various power states.

\begin{tabular}{|c|c|}
\hline Power state & Power consumption \\
\hline Sleeping, with sleep clock active & $1.5 \mu \mathrm{W}$ \\
\hline MCU active at 32MHz, low activity & $29 \mathrm{~mW}$ \\
\hline MCU active at 32MHz, high activity & $37 \mathrm{~mW}$ \\
\hline MCU active, transmitting/receiving & $81 \mathrm{~mW}$ \\
\hline
\end{tabular}

Table 1: Typical power consumptions of CC2430 in various power states ${ }^{3}$

Assuming a $0.1 \%$ duty cycle (with the node sleeping $99.9 \%$ of the time, and active for the remaining period including data transmission), the node will consume an average of $82 \mu \mathrm{W}$ (plus any power consumed by sensing and data acquisition hardware). Operating from two standard AA-sized alkaline batteries, the node can be 
expected to operate for in excess of a year. When powered from harvested energy, its life can potentially be extended indefinitely - with the added benefit of being able to recharge after total depletion.

\section{HARVESTING AND STORAGE DEVICES}

Energy harvesting is a means to extend the lifetime of the autonomous sensor node beyond that of a primary battery. The dominant energy harvesting technologies, of use to autonomous sensors, are:

1. Photovoltaics (producing electricity from ambient light - either indoors or outdoors)

2. Vibration (producing electricity from vibrations of the surface the sensor is deployed on)

3. Thermoelectrics (producing electricity from a temperature gradient)

In order to compare the effectiveness of the differing technologies, we consider the deployment of an autonomous sensor in a machinery monitoring application. For example, a small compressor pump in a factory lit by a mix of natural and artificial light may vibrate at a mains frequency of $50 \mathrm{~Hz}$ and an amplitude of 50 milli$g$ RMS (where $g$ corresponds to an acceleration of $9.81 \mathrm{~ms}^{-2}$ ). It may also be exposed to an average lighting level of up to 1000 lux and have an exploitable temperature gradient between its casing and the surrounding air of approximately $35^{\circ} \mathrm{C}$.

Of the three main technologies, photovoltaics have been by far the most prevalent for powering autonomous sensors. Photovoltaic cells produce electricity from photons by means of a semiconductor p-n junction. This technology is at a relatively advanced stage of development, and many deployment situations for autonomous sensors will be lit by natural or artificial lighting. In the light levels described, a Schott Solar OEM 1116929 amorphous silicon photovoltaic module (ASi photovoltaic cells are generally more efficient at lower light levels such as this) can be expected to produce a nominal power of $1.79 \mathrm{~mW}$ at 3.33 volts $^{5}$.

Thermoelectric energy harvesters exploit the Seebeck effect, in which electricity is generated from a temperature difference across a thermocouple. In general, thermoelectric devices require a large and sustained temperature gradient between two surfaces in order to provide useful power. A Micropelt TE-Power-Plus module produces approximately $560 \mu \mathrm{W}$ of stabilised power from a source temperature of $60^{\circ} \mathrm{C}$ and ambient temperature of $25^{\circ} \mathrm{C}^{6}$.

Vibration energy harvesters use one of three mechanisms to convert vibration to electricity: electromagnetic, piezoelectric or electrostatic. Electromagnetic generators use a resonant magnet and coil arrangement to generate electricity, whereas piezoelectric-based generators use a piezoelectric resonant beam which generates electricity when subjected to strain. Electrostatic generators exploit capacitive effects but, due to machining and practical issues, have not become widespread. Vibration energy harvesters are sensitive to the frequency of vibrations of a surface, and their deployments are generally limited to machinery that vibrates at a constrained range of frequencies and amplitudes. For the vibration levels specified, the PMG17 microgenerator from PMG Perpetuum will produce a power output of $1.5 \mathrm{~mW}$, rectifying up to 4 volts ${ }^{7}$.

One must take into account the availability of environmental energy when selecting a harvesting device. Photovoltaic modules are a good solution, but are susceptible to being obscured over time by dust and dirt, which may present a major problem in an industrial environment. Vibration energy harvesters do not suffer from such problems, but are sensitive to vibration frequencies (and, unlike photovoltaics, they rely on the machinery being operational in order to provide power to the autonomous sensor). In a similar way, thermoelectrics depend on good thermal contact with the device, and the required temperature difference being present between two surfaces.

\section{EXISTING ENERGY HARVESTING SYSTEMS}

A number of projects have used energy harvesting technologies to deliver sustainable power for autonomous sensors. Photovoltaic energy harvesting is by far the most prevalent form of technology - in part due to the plentiful supply of light in many deployment settings, and the low cost of photovoltaic modules. Nodes conventionally store electrical energy in supercapacitors or batteries to achieve operation in darkness and during 
bursts of high current draw. A notably sophisticated solar energy harvesting platform, Prometheus, buffers energy both in supercapacitors and a lithium polymer rechargeable battery ${ }^{8}$. The supercapacitor is used for short-term energy storage, while the battery stores excess energy and is used to top up the supercapacitor when it becomes depleted (for example, overnight during darker winter months). In this way, stress on the battery is minimised (supercapacitors are far less sensitive to repeated charge/discharge cycling than batteries), and the system could be expected to last longer than simpler systems such as Heliomote (which buffers energy only in batteries).

Several prototype systems incorporating vibration energy harvesting have been developed. For example, the $S^{5} \mathrm{NAP}$ uses a commercially-available electromagnetic vibration energy harvester to power an accelerometerbased condition monitoring system ${ }^{10}$. Additionally, a microgenerator with a volume of less than $1 \mathrm{~cm}^{3}$ has been developed in conjunction with a custom sensor node and is described later in this issue ${ }^{11}$. In both systems, energy harvested from vibrations is buffered in supercapacitors to permit nodes to draw large bursts of power during radio transmissions and sensing operations.

Relatively few sensor node deployments based on thermoelectric energy harvesting have been reported. Typically, such deployments have depended on a very large temperature gradient being present in order to deliver sufficient levels of energy; for example, a recent investigation successfully deployed a wireless sensor network on aluminium smelters ${ }^{12}$. Further developments in thermoelectric energy harvesting devices have substantially increased their efficiencies. EnOcean have developed the ECT100 thermal energy harvester kit, which incorporates a thermoelectric energy harvester and radio transmitter module ${ }^{13}$.

\section{A DEVELOPED SYSTEM: HARDWARE}

A significant challenge in maximising the power harvested from indoor lighting is in ensuring that power conditioning circuitry consumes a minimal amount of power whilst obtaining near-optimal power levels from the photovoltaic module. Systems developed for outdoor deployment consume relatively large levels of quiescent power - in many cases more than is generated by amorphous silicon module described earlier. Outdoors it is necessary to 'track' the maximum power point voltage of the photovoltaic module (which changes with varying light level), as the intensity of outdoor light may vary between darkness and tens of thousands of lux and it is important for modules to operate efficiently in this wide variety of illumination. In outdoor situations, maximum power point tracking can yield up to a $30 \%$ improvement in efficiency compared to operating the module at a fixed voltage.

In typical indoor scenarios, however, light levels from artificial lighting rarely exceed 1000 lux. At 200 lux, the module described will operate optimally at 3.01 volts, while at 1000 lux it operates at 3.33 volts. It is acceptable in this case to operate at a fixed voltage between the minimum and maximum operating voltages expected in the deployment situation. From real-world experiments, we have determined that by operating the module at 3.10 volts, the minimum efficiency at this range of illuminations is in the region of $97 \%$ (this is the raw power generated from the module compared to the power it would generate when operated at its maximum power point). Active tracking of the maximum power point voltage would be likely to consume more power and thus reduce the converter efficiency.

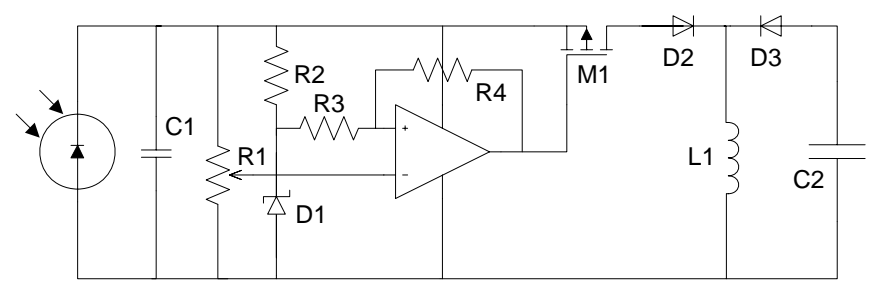

Figure 2: Circuit diagram from the developed system

A circuit diagram of the developed system is shown in Figure 2. A small tantalum electrolytic capacitor, C1, acts as a temporary buffer from the photovoltaic module. A large potentiometer, R1, divides the supply voltage 
and feeds it into the inverting input to the comparator. This permits the operating voltage of the photovoltaic module to be trimmed. The non-inverting input to the comparator is provided by a voltage reference chip, D1, with some additional hysteresis circuitry from the comparator output. As the supply voltage gradually rises, in our case past 3.1 volts, the comparator output is driven low, which turns on the MOSFET. Energy is fed into the output part of the circuit, causing the supply voltage to drop and the comparator output to be driven high.

The output part of the circuit is comprised of an inductor, diodes, and supercapacitor, C2. This, together with the MOSFET and its control circuitry, forms a modified buck-boost converter. Through this arrangement, the source voltage can be kept isolated from the supercapacitor voltage, meaning that the photovoltaic module can be operated at a constant voltage. This is in contrast to conventional switching converters, which operate to maintain a constant voltage on their output. An additional diode is required to prevent the capacitor from discharging back through the supply rail while the photovoltaic module is in darkness. An important detail about this circuit is that the polarity of the output is opposite to the supply. All components used in this circuit were chosen for their high efficiency and low quiescent power consumption.

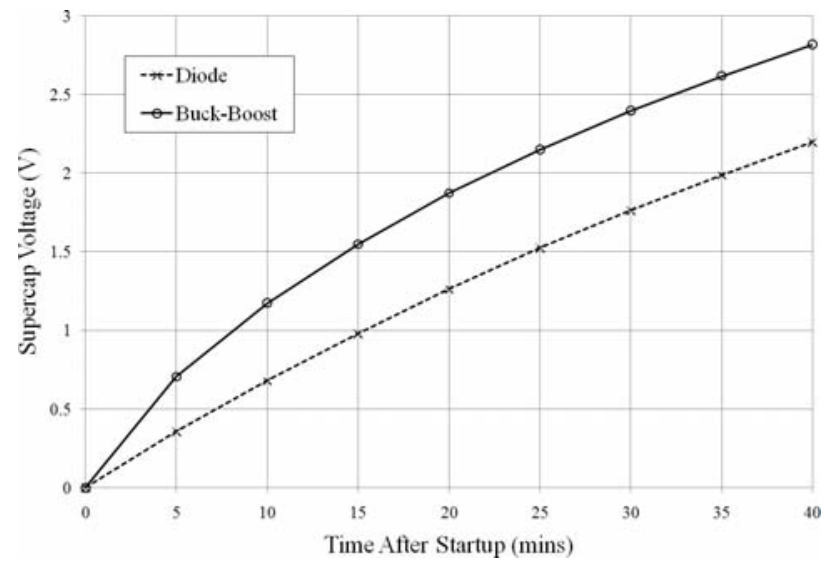

Figure 3: Charging profiles for 0.5F supercapacitor with diode and buck-boost converter

Figure 3 shows the charging profile of this circuit, compared to the performance of a capacitor simply charging through a diode. These tests were performed in a typical office environment under a light level of approximately 1000 lux. A typical turn-on voltage for autonomous sensor nodes is 2.0 volts, and it can be seen from the figure that the switching circuit is substantially faster at reaching this voltage than the single-diode circuit. The comparative benefits, though, become less significant at low light levels.

\section{A DEVELOPED SYSTEM: EMBEDDED SOFTWARE}

Modern wireless autonomous sensors have evolved to contain significant onboard functionality over and above communications, including sensor processing, energy management, actuation and locationing. The embedded software is often implemented in the application layer of the communications stack, resulting in an unstructured, top-heavy and confusing stack. The developed system provides all major node functions with the benefits of a structured, layered architecture; these include the structured planning of a modular architecture, allowing efficient code reuse, and promoting the development and standardisation of interchangeable protocols.

The node's software structure is built around a unified framework, developed as part of this research. The framework defines a "basic template stack", from which "interface stacks" are derived. Several of these interface stacks (each implementing individual and distinct functionality) are connected together via a shared application layer, thus forming a unified stack (see Figure 4 for the unified stack used in the developed system, which structures communications, energy management and sensor processing). Use of this framework extends the benefits of a structured, layered model (that have, for decades, been appreciated by the communications stack) to all major node functions. These benefits include promoting modular design, permitting efficient code reuse, and aiding the development and standardisation of interchangeable protocols. 


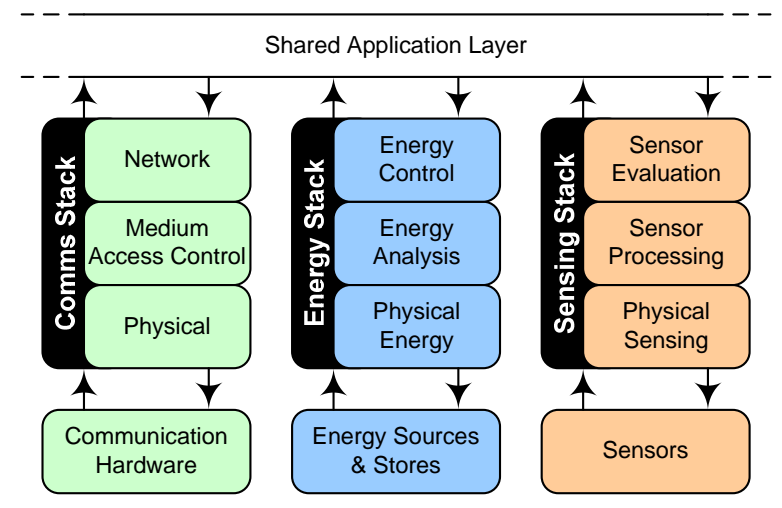

Figure 4: A unified stack, to structure the multiple interfaces on the sensor node

In the developed system, the communication system is the TI Simple Packet Protocol, with minor modifications in order to integrate it into the unified stack. The energy stack is responsible for the node's energy management, including controlling and monitoring the energy sources and stores. The energy stack is divided into three layers:

- Physical Energy Layer (PYE): interfaces with the energy store(s) to obtain voltage readings, which are provided to the EAN. In the developed system, the PYE monitors the voltage across the supercapacitor store. In the future, this layer will perform physical switching in order to direct the flow of energy in a multiple store/source node, and also monitor the yields from energy harvesting sources.

- Energy Analysis Layer (EAN): accepts data from the PYE and provides 'interpreted' data on the energy stores to the ECO. In the developed system, the EAN converts the store voltage to a residual energy (using a store model), and provides the ECO with an estimation of the 'remaining usable energy', and 'remaining lifetime'. In the future, this layer will provide additional energy information, including the rate and frequency of energy generation and energy usage.

- Energy Control Layer (ECO): takes a high-level view of the energy subsystem, reporting to the shared application layer on the energy status and sustainability of the node in a hardware-independent format. In the developed system, the ECO evaluates the 'remaining usable energy' estimation to allocate an Energy Priority $(\mathrm{EP})^{14}$. In the future, this layer will make decisions about the components of the energy subsystem, controlling the transfer of charge between energy stores and sources at a high level.

The sensing stack is responsible for notifying the shared application layer of detected events in the sensed environment, along with performing data correction and uncertainty analysis. The sensing stack is divided into three layers:

- Physical Sensing Layer (PYS): interfaces with hardware in order to provide raw sensor data to the SPR, while masking the complexities involved in obtaining the sensor reading. In the developed system, the PYS activates a temperature sensor and ADC, and obtains voltage readings from the sensor.

- Sensor Processing Layer (SPR): accepts raw sensor data from the PYS, and performs preliminary processing, providing 'interpreted' data to the SEV. In the developed system, the SPR scales and offsets the raw sensor reading in order to convert the voltage into a temperature reading. In the future, this layer will include localised data fusion, for example by fusing pressure sensor data with temperature data in order to provide temperature compensation. It will also use an embedded sensor model to provide the SEV with error bars, estimations of drift and uncertainty, and indications of sensor faults.

- Sensor Evaluation Layer (SEV): evaluates data from the SPR, making high level decisions, observations and corrections. In the developed system, the SEV applies Rule Managed Reporting ${ }^{14}$ in order to provide the shared application layer with details of detected events. 
Through the use of this architecture, embedded software has been developed in a structured fashion. Functionality has been split into logical blocks, which can be easily modified without affecting the surrounding layers.

\section{CONCLUSIONS}

This paper has highlighted wireless autonomous sensing as an attractive technology that, due to its economic benefits, is likely to continue to receive considerable interest. A variety of low-power autonomous sensing platforms are now commercially available and, due to recent advances in energy harvesting technology, it is becoming viable to replace the on-board battery with such a source. A system has been developed which uses a $0.5 F$ supercapacitor as its sole energy store. The supercapacitor is efficiently charged via a photovoltaic module illuminated by fluorescent lighting in a regular office building. The embedded software in the developed system was structured using a "unified framework", which specifies and structures the communications, energy management and sensor processing interfaces on the node. This promotes a structured and modular design, and hence allows for efficient code reuse and encourages the standardization of interchangeable protocols.

\section{REFERENCES}

[1] J.M. Rabaey, M.J. Ammer, J.L. Da Silva, Jr., D. Patel, and S. Roundy, "PicoRadio supports ad hoc ultra-low power wireless networking, " IEEE Computer, vol. 33, pp. 42-48, 2000.

[2] D.K. Arvind, "Wireless Sensor Networks - A Mission To The USA," Department of Trade and Industry Global Watch Seminar, London, November 2005.

[3] “A True System-on-Chip solution for 2.4 GHz IEEE 802.15.4 / ZigBee(TM) (Rev. F), ’Texas Instruments, Dallas, TX, 2007.

[4] “MRF24J40 data sheet,” Microchip Technology Inc., Chandler, AZ, 2006.

[5] “ASI-OEM Solarmodules for Indoor, ” Schott Solar GmbH, Putzbrunn, Germany, 2006.

[6] “Thin film thermogenerators and sensing devices,” Micropelt GmbH, Freiburg, Germany, 2007.

[7] “PMG17 vibration energy harvesters, ” PMG Perpetuum Ltd., Southampton, UK, 2008.

[8] X. Jiang, J. Polastre and D. Culler, "Perpetual Environmentally Powered Sensor Networks, " presented at 4th Int'l Conf. Information Processing in Sensor Networks/Special Track on Platform Tools and Design Methods for Network Embedded Sensors (IPSN/SPOTS), Los Angeles, CA, 2005.

[9] V. Raghunathan, A. Kansal, J. Hsu, J. Friedman and M. Srivastava, “Design considerations for solar energy harvesting wireless embedded systems," in Proceedings of the 2005 Fourth International Symposium on Information Processing in Sensor Networks, 2005, pp. 457-62

[10] S. George, "Development of a Vibration-Powered Wireless Temperature Sensor and Accelerometer for Health Monitoring,” presented at 2006 IEEE Aerospace Conference, Big Sky, MT, 2006.

[11] R.N. Torah, P. Glynne-Jones, M.J. Tudor and S.P. Beeby, "Energy Aware Wireless Microsystem Powered by Vibration Energy Harvesting," presented at PowerMEMS 2007, Freiburg, Germany, 2007.

[12] M. Schneider, J. Evans, P. Wright and D. Ziegler, "Designing a thermoelectrically powered wireless sensor network for monitoring aluminium smelters," Proceedings of the Institution of Mechanical Engineers, Part E: Journal of Process Mechanical Engineering, 2006, vol. 220, pp. 181-90

[13] “Thermal energy harvester kit ECT 100 perpetuum”, EnOcean GmbH, Oberhaching, Germany, 2007.

[14] G.V. Merrett, N.R. Harris, B.M. Al-Hashimi, and N.M. White, "Energy Managed Reporting for Wireless Sensor Networks," Sensors and Actuators A: Physical, vol. 142, pp. 379-389, 2008. 